\title{
Influence of Vertical Resolution on the Validation of Atmospheric Chemistry Instruments
}

\author{
Guochang Zhang \\ Department of Physics and Information Engineering, Shangqiu Normal University, Shangqiu, China \\ E-mail: zhangguochang1@gmail.com \\ Received March 5, 2011; revised April 22, 2011; accepted May 1, 2011
}

\begin{abstract}
A large number of valida tion cam paigns for atm ospheric che mistry instrum ents are being carried out and more such st udies will be performed in the future. The aims of validation are to confirm the accuracy and precision of the measurement of a new instrument. There are many factors that may deteriorate the validation results and one of them is the vertical resolution of instruments when using the profiles intercomparison approach. The influence from the vertical resolution can be eliminated by using the averaging kernel method but it is necessary to find the conditions for using the method. This study simulated the influence of vertical resolution for a cert ain curvature. The results show that both the curvature of a profile and th e difference of vertical resolution between two instruments have pos itive correlation with the differences between their measurements. The quanti tative estim ations of influ ence for some practical vertical resolu tions were obtained. The combined error of two instruments was defined as the criteria to judge the significance of influence. A c ase study based on the sim ulated results was demonstrated to show wh en the influence from the vertical resolution should be considered and when such influence can be omitted in order to avoid some unnecessary works in validation.
\end{abstract}

Keywords: Simulation, Validation, Vertical Resolution, Atmospheric Chemistry Instrument

\section{Introduction}

Limb-scanning remote-sounding atmospheric chemistry instruments onboard satellit es are w idely used $t$ o measure atmospheric parameters like the density of $g$ ases, temperature and pressu re at different altitudes, thus forming profiles of par ameters. For the validation of a new rem ote sounder, it is necessary to compare its measurements with the observ ations of o ther proved instruments at the same time and location. There are many factors that can deteriorate the validation results, for example, measuring different air masses be cause of a tmospheric fluidity, the che mical reaction in the atmo sphere, the different char acteristics of instruments like the vertical and horizontal resolution, etc. $[1,2]$. The ver tical $r$ esolution shoul $d$ be especially concerned during the validation for limb-scan remote sounders which usually present their output data with profile form of atmospheric parameters. Figure 1 shows an intercomparison of $t$ emperature prof iles d uring the $\mathrm{v}$ alidation for M IPAS/ENVISAT, Michelson Inte rferometer for Pas sive Atmospheric Sounding aboard the Environmental Satellite of European Space Agen cy [3,4] (this instrument is named
MIPAS-E hereafter) by using the data from MIPAS-B - a remote sounder $t$ hat is si milar to M IPAS-E but aboard a large Balloo $\mathrm{n}$ [5-7]. Bo th instruments adopted limb-sounding geo metry and can $m$ easure tens of atmospheric par ameters prof iles like pressure and tem perature profiles, mixing volume ratio profi les of $\mathrm{O}_{3}, \mathrm{H}_{2} \mathrm{O}, \mathrm{HNO}_{3}$, $\mathrm{N}_{2} \mathrm{O}, \mathrm{CH}_{4}$ and $\mathrm{NO}_{2}$ etc. within a short period of time. In the figure, $t$ he $r$ ight pan el $g$ ives the temperature profile of MIPAS-E with vertical resolution $3 \mathrm{k} \mathrm{m}$, the profile of the same parameter from MIPAS-B but with vertical resolution $1 \mathrm{~km}$ which is the nominal resolution of the instrument. An additional $r$ etrieved temperature profile fro $m$ MIPAS-B with vertical resolution $3 \mathrm{~km}$ was also presented. The left panel $g$ ives the differences of profi les bet ween MIPAS-E and MIPAS-B with vertical resolution $3 \mathrm{k} \mathrm{m}, 1 \mathrm{~km}$ and its smoothed profile (details in section 3.3), resp ectively. The agreements between $\mathrm{t}$ he MIPAS-E and M IPAS-B profiles are generally good in the whole altitude range of comparison. However, in the range of $169-126 \mathrm{hPa}(11-15 \mathrm{~km})$, the $d$ ifferences are obvi ously larger when $\mathrm{u}$ sing the MIPAS-B profile with vertical re solution $1 \mathrm{~km}$ than the differences when using another MIPAS-B profile w ith verti- 


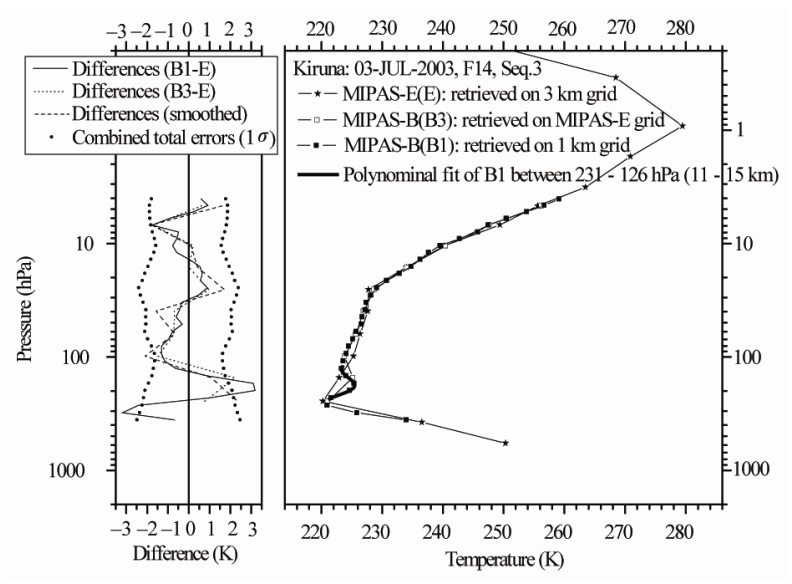

Figure 1. Intercomparison of temperature profiles between MIPAS/ENVISAT (MIPAS-E) and MIPAS-B [13] (Zhang, 2006).

cal resolution $3 \mathrm{~km}$. This indicates that if the vertical resolution of an instrument used for validation is different from that of the instrument to be validated, the validation results may be incorrect. Ther efore, the ver tical resol ution of all instruments that involved in validation should be equal in principle. How ever, because of the limitation of char acteristics of each instrument, this requi rement will not be al ways sa tisfied. In th is c ase, for el iminating the influence due to ver tical reso lution, the method usi ng aver ageing kernel matrix to smooth the profile with fine vertical resolution may be performed $[8,9]$. Howev er, so metimes this step will not be carried out if the influence can be ignored based on empirical knowledge of validation [10-12]. However, this kind of empirical judgment is not always correct. Hence, it is worth to evaluate the condition that what difference of vertical resolution between the instruments involved in validation is acceptable or unacceptable since it is beneficial to sci entist for redu cing the computational burden, financial cost and saving time in validation.

\section{Reasons of Vertical Resolution Influencing Validation}

In general, the atmospheric parameters like temperature and density of gases are variables with respect to altitude. As a re sult, the profiles of a tmospheric pa rameters are smoothly continuous curves which have different curvature at different level of altitu de. However, in struments can only measure atmospheric parameters at certain altitude levels. Then the measured profiles form the broken lines as the temperature profiles shown in Figure 1. Vertical resolutions may be ten s of meters (in situ measurement), several kilometers (limb-viewing remote sensing), and more than ten kilometers (nadir remote sensing).

In the valid ation when u sing a m ethod of profiles in - tercomparison, the first step should be to in terpolate all the profiles onto a defined vertical grid (represented by altitude levels or pressure levels) b y using logarithm, or linear, or $\mathrm{sp}$ line algo rithms. The in terpolation algorith $\mathrm{m}$ may introduce extra errors to the intercomparison and the errors have positive correlation to the difference of vertical resolution am ong the profiles. This is because that the profile which has ro ugh $\mathrm{v}$ ertical $\mathrm{r}$ esolution can no $\mathrm{t}$ resolve the fine structure of atmospheric parameter field. Obviously, if the profiles are straigh t lines, the ex tra errors disappear an $d$ the vertical res olution has no influence on intercomparisons. Therefore, in order to evaluate the e rrors i ntroduced by the v ertical grid, two factors should be considered simultaneously the vertical resolution and the curvature of profile.

\section{Simulation and Results Analysis}

\subsection{Curvature in a Profile}

In general, there are many different values of curvature for a profile of atm ospheric parameters. See Figure 1, for the MIPAS-B measured temperature profile at $1 \mathrm{~km}$ vertical grid, between the height region $126-231 \mathrm{hPa}$, the profile has relative large curvatures, i.e. small radii of curva ture co mparing with the seg ments which are nearly straight lines in regions of $360-268 \mathrm{hPa}, 109$ $28.6 \mathrm{hPa}$ and $25-4 \mathrm{hPa}$. For perfor ming simulation and assuming a segment of a profile, which represents the true values of an atmospheric parameter at different altitude levels, has a radi us of curvat ure $r$. The arb itrary unit of atmospheric parameter is used without incur any wrong conclusion. Small $r$ rep resents that the profile has fine structure. Further, it is assumed that all instruments that adop $t$ di fferent ver tical grid measure the true value of atmospheric parameters. Some of atmospheric chemistry instruments used to adopt one of the following vertical resolutions $[0.5,1,2,3,4,5,6]$ $\mathrm{km}$. The choosed curvature radius of the profile for simulation should not be less than $1 \mathrm{k} \mathrm{m}$ in order to ensure that at least two points can be ex tracted from the profile. In order to ensure $t$ hat the simulation can be carried out for all the verti cal reso lutions just mentioned above, we choose a curve with a curv ature radius $4 \mathrm{k} \mathrm{m}$ to represent the true profil e of an atm ospheric parameter.

\subsection{The Profile Number for Intercomparison}

For validation of instruments, a definite conclusion should depend on statistical results of intercomparisons. Figure 2 gi ves the si mulation res ults fo $r$ the i nfluence of $t$ he number of profiles i nvolved in i ntercomparison t o the 


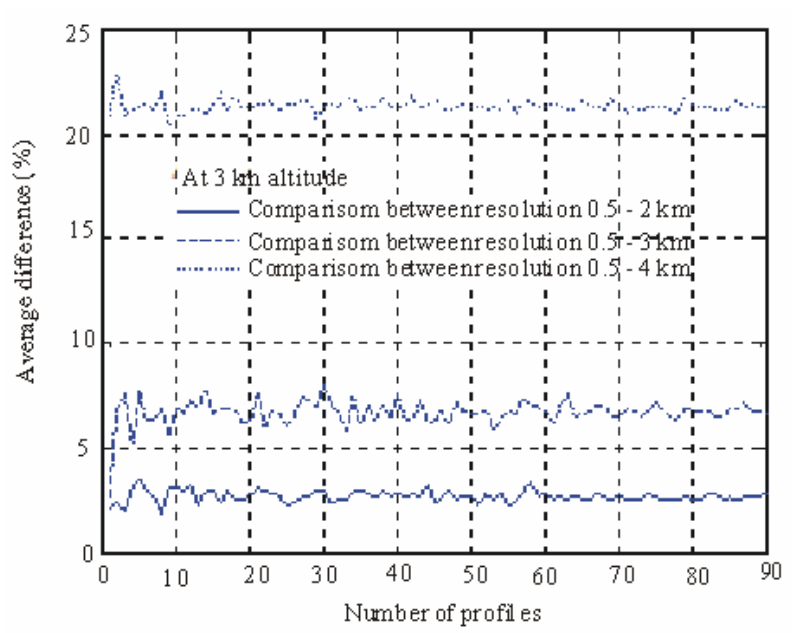

Figure 2. Influence of the profile number to the comparison between profiles with different vertical resolution.

comparison results, i.e. the measurement difference of a proved instrument and a $\mathrm{n}$ unproved one. It is cl ear that the differences vary with the $\mathrm{n}$ umber of $\mathrm{p}$ rofiles es pecially when the number is less than 20 . However, the differences approach a constant with the increasing of profile number. This actually indicates the changing trend of the standard error of comparison with the number of intercomparisons. Here, 70 simu lated profiles will be used during the simulation comparisons.

\subsection{Simulation Comparison}

The sim ulation procedure inclu des the fo llowing step s. Firstly, let the cu rve $x=\sqrt{r^{2}-(h-r)^{2}} \quad(0 \leq h \leq 2 r)$ represents the true profile of an atm ospheric parameter. Here, $h$ is the altitude, and $r$ is the cu rvature radius. Parameter $x$ has an arbitrary unit. Secondly, for each vertical grid given above, 70 profiles were $p$ roduced by extracting a point from the true profile at each level of altitude. These 70 simulated profiles represent the measured profiles of an instrument without any errors. Thirdly, for each comparison, $t$ he values of $t$ he parameter at eac $h$ level of altitude were calculated by interpol ating all the profiles linearly into the vertical grid with the vertical resolution $0.5 \mathrm{~km}$. Finally, the calculations for the a verage difference at each level of altitude in the comparison for the two kinds of p rofile were carried out. For eliminating the influence of the absolute value of parameter to conclusions, the a verage difference i n percentage wa $\mathrm{s}$ used. The standard deviations of each average were also calculated to denote the dispersing extent of average difference.

There a re $\mathrm{m}$ any different $\mathrm{c}$ ombinations of $\mathrm{i}$ ntercomparison according to the given vertical resolutions. Here, the vertical resolutions w hich are fre quently appeared during practical validation activities were considered. Figure 3 shows t he simulation results for the com parisons between vertical resolutions of $0.5-1 \mathrm{~km}, 0.5-2$ $\mathrm{km}, 0.5-3 \mathrm{~km}, 0.5-4 \mathrm{~km}, 0.5-5 \mathrm{~km}, 0.5-6 \mathrm{~km}$, and $1-$ $3 \mathrm{~km}$. The results clearly show s that the vertical resolution of instruments has influence on intercomparison, i.e. the differences of com parisons have positive correlation with the diffe rence of verti cal resolution betwee $n$ two kinds of pro files even if both in struments measured the true value of atmospheric parameters. The averaged standard deviation bars in dicate that with inc reasing of difference of vertical resolution the precision of comparison decreases. This may lead to an underestimation of precision of an in strument. Even if the dif ference of vertical resolution is equal for each comparison (Figure 4), the influence to comparison is not the same. This is because the influence of vertical resolution is related with the curvature of the true profile. For a fixed curvature, the larger vertical res olution of instr uments incurs larger differences between their measurements.

In fact, the true profile of any atmospheric parameter is unavailable. Fortunately, for most of the cases of validation, the profiles from th ose proved in struments have finer ve rtical resol ution tha $n$ the profiles from the instruments which need to be validated. Thus, these profiles with fine vertical resolution can be regarded as true profiles. The curvature of a profile can be deduced from the fitted curve of the profile. Generally, the curvature varies with point of the fitted curve. Theref ore, the curvature of the c urve (or a segment of curve) needs to be determined? For estim ating the $\mathrm{i}$ nfluence of vertical resolution, th e a veraged c urvature of th e cur ve (or a segment of a curve) can be regarded as the curvature of the whole curve. In Figure 1, the fitted curve of MIPAS$\mathrm{B}$ te mperature profile in the $\mathrm{r}$ ange of $11-15 \mathrm{~km}$ is a polynomial curve. And the averaged curvature radius of the curve around the peak point is about $1 \mathrm{~km}$.

The criteria whether the in fluence of ve rtical res olution can be omitted depend on the measurement errors of two instruments which are in volved in inter comparison. Assuming two instruments have measurement errors $\sigma_{1}$, $\sigma_{2}$, respectively, the combined error is

$$
\sigma=\sqrt{\sigma_{1}^{2}+\sigma_{2}^{2}} .
$$

If the differences of simulated c omparison are lar ger than the combined errors, then the in fluence of vertical resolution to validation s hould not be neglected. Figure 3 shows that the differences between profiles of vertical resolution $1 \mathrm{~km}$ and $3 \mathrm{~km}$ are $2-16 \%$ when the curvature radius is $4 \mathrm{~km}$. This is ju st the case in term s of vertical resolution for the validation of MIPAS-E by using MIPAS-B data. In the range of $11-15 \mathrm{~km}$, the maximum of $t$ he $\mathrm{c}$ ombined e rror of te mperature di fferences be - 
tween MIPAS-E a nd M IPAS-B is $1.7 \%$. Since the curvature radius around the peak point of the fitted curve is about $1 \mathrm{~km}$, the simulation can not be carried out because the inter polation al gorithm is invalid for the M PAS-E profile which has a vertical resolution of $3 \mathrm{~km}(>1 \mathrm{~km})$. However, it is clear that the simulated differences in this case will be la rger than 2 - $16 \%$ because the curvature radius $1 \mathrm{~km}$ is less than $4 \mathrm{~km}$ and it is de finitely larger than the $\mathrm{m}$ aximum of the $\mathrm{c}$ ombined e rror $1.7 \%$. Therefore, in the range of $11-15 \mathrm{~km}$, the results of direct comparison between $t$ he $m$ easurements of M IPAS-E a nd MIPAS-B (Figure 1) doesn't give the true differences of th e m easurements betwee $\mathrm{n}$ the tw o inst ruments, i.e. the differences of comparison in $11-15 \mathrm{~km}$ may include significant co ntribution f rom the influen ce of vertical resolution. Therefore, for this case the in fluence of vertical resolution should be considered. For the segments

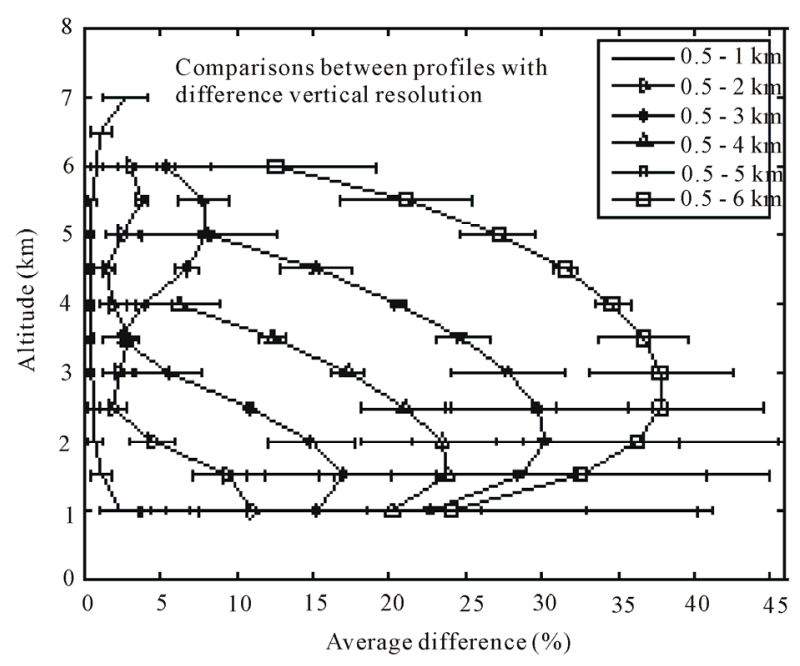

Figure 3. Simulated comparisons between two profiles with different vertical resolution.

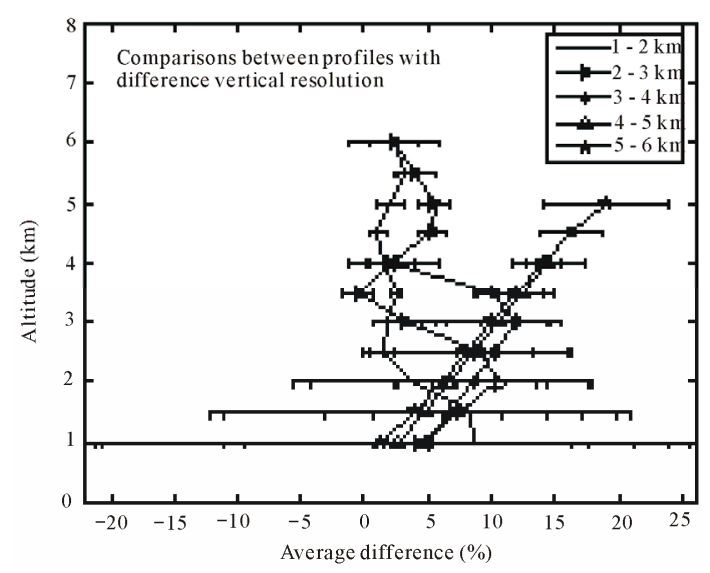

Figure 4. Simulated comparisons between two profiles with different vertical resolution where the difference of vertical resolution is equal for each comparison. of temperature profiles above $15 \mathrm{~km}$ and below $11 \mathrm{~km}$, since their c urvatures are very sm all, the influe nce of vertical resolution can be omitted.

If the influence of vertical resolution can not be omitted and there fore direct comparison is unacceptable, the following two ap proaches for improving validation can be adopted. One is to retrieve the profiles of atmospheric parameter with the sam e vertical resolution for both instruments. As an example, the result fr om this kind of approach is shown in Figure 1. This approach, however, is often li mited by the characteristics of instrum ent, retrieval algorithm, etc., and not always feasible. The second approach is the $\mathrm{m}$ ethod to use the averaging kernel. This ap proach was d escribed by Rodg ers and C onnor [14]. B efore performing th e com parison, the $\mathrm{p}$ rofiles with higher vertical resol ution need to be smoothed. If disregarding noise, the retrieved profile $X_{r e}$ is a wei ghted average of the "true" profile $X_{\text {true }}$ and the a priori profile $X_{a}$ in the form of

$$
X_{r e}=A X_{\text {true }}+(I-A) X_{a},
$$

where $A$ is th e a veraging kernel $\mathrm{m}$ atrix and $I$ de notes the i dentity matrix. Th e higher-resolved profiles $X_{B}$ of instrument $B$ are smoothed by applying the av eraging kernel matrix of the low-resol ved profiles $X_{E}$ of instrument $\mathrm{E}$. And the profile smoothing is done by

$$
\tilde{X}_{B}=A_{E} X_{B}+\left(I-A_{E}\right) X_{E a},
$$

where $X_{E a}$ denotes the a priori profile of in strument E. Comparing equation (3) with equation (2), it is clear that $\tilde{X}_{B}$ is the res ult derived from the instr ument $E$ inve rse mode, if $X_{B}$ is assumed to be the true profile. Thus, in the difference of $\tilde{X}_{B}-X_{E}$ the contributions originating from different vertical resolution are reduced. Figure $\mathbf{1}$ als o presents the difference profile between smoothed MIPAS-B temperature and MIPAS-E measurements (for clarity, the sm oothed MIPAS-B tem perature profile was not plotted). Above $15 \mathrm{~km}$ and below $11 \mathrm{~km}$, the difference profile is very close to the one of direct comparison. This indicates that $\mathrm{t}$ he vertical res olution influences on the validation are very small and consistent with the simulation results mentioned here. However, the improvement of com parison between altitudes $11-15 \mathrm{~km}$ is obvious. It indicates that a fter adopting the averaging kernel approach to smoothing the higher-resolved MIPAS-B profile, the i nfluence of vertical resol ution to the comparisons was reduced.

\section{Conclusions and Outlook}

For the validation of a $\mathrm{n}$ atm ospheric c hemistry instru ment by comparison with the p rofiles from prove $\mathrm{d}$ instruments, vertical res olution of p rofiles can deteriorate 
the validation results. The quantitative simulation results show the extent of deterioration due to the difference of vertical res olution bet ween the profiles for comparison for a certain c urvature of profile. The results also show that when the difference of vertical resolution is equal for each pair of $\mathrm{c}$ omparison, the larger ve rtical resolutions have more influence on comparison for a given curvature. The influence of vertical resolution on a validation has to be considered if its caused difference is beyond the combined errors of two instruments. In general, this kind of influence can be eliminated or reduced by using the averaging kernel of profile with rough vertical resolution to smooth the profile with fine vertical resolution.

Since the number of validation activities will increase in the future, it is useful to simulate the influence of vertical resolution on validation for different combinations of vertical resolution and d ifferent curvatures of profile. Our ne $\mathrm{xt}$ aim is to establish a datab ase based o $\mathrm{n}$ the complete simulation results. The database will be freely accessible for all scientists engaging in validation.

\section{References}

[1] C. D. Rodgers, "Inverse Methods for Atm ospheric Sounding: Theory and Practice," World Scien tific Press, Singapore, 2000. doi:10.1142/9789812813718

[2] T. von C larmann, "Valid ation of Remotely Sen sed Profiles of Atm ospheric State Variables: Strategies and T erminology," Atmospheric Chemistry and Physics, Vol . 6, 2006, pp. 4311-4320. doi:10.5194/acp-6-4311-2006

[3] H. Fischer and H. Oelhaf, "R emote Sensing of Vertical Profiles of Atm ospheric Tr ace Constituents with Mipas Limb-Emission Spectrometers," Applied Optics, Vol. 35, No. 16, 1996, pp. 2787-2796. doi:10.1364/AO.35.002787

[4] H. Fischer, M, Birk, C. Blom, et al, "MIPAS: An I nstrument for Atmospheric and Climate Research," Atmospheric Chemistry and Physics, Vol. 8, 2008, pp. 2151-2188. doi:10.5194/acp-8-2151-2008

[5] F. Friedl-Vallon, G. Maucher, M. Seefeldner, et al., "Design and $\mathrm{Ch}$ aracterization of the Balloon-Borne Michel- son Interferometer for Pa ssive Atmospheric Sounding (MIPAS-B2)," Applied Optics, Vol. 43, No. 16, 2004, pp. 3335-3355. doi:10.1364/AO.43.003335

[6] A. Kleinert, "Correction of Detector Nonlin earity for the Balloon-Borne Michelson Interferometer for Passive Atmospheric Sounding," Applied Optics, Vol. 45, No. 3, 2006, pp. 425-431. doi:10.1364/AO.45.000425

[7] A. Kleinert and O. Trieschm ann, "Phase deter mination for a Fourier Tr ansform Infrared Spectrometer in Emission Mode," Applied Optics, Vol. 46, No. 12, 2007, pp. 2307-2319. doi:10.1364/AO.46.002307

[8] H. M. Worden, J. A. Logan, J. R. Worden, et al., "Comparisons of Tro pospheric Emission Spectrometer (TES) ozone Profi les to Ozonesondes: Methods and I nitial Results," Journal of Geophysical Research Atmospheres, Vol. 112, 2007. doi:10.1029/2006JD007258.

[9] E. Mahieu, P. Duchatelet, P. Demoulin, et al., "Validation of ACE-FTS v2.2 Measurements of $\mathrm{HCl}, \mathrm{HF}, \mathrm{CCl}_{3} \mathrm{~F}$ and $\mathrm{CCl}_{2} \mathrm{~F}_{2}$ Using Space-, Balloon- and Groun d-Based Instrument Observations," Atmospheric Chemistry and Physics, Vol. 8, 2008, pp. 6199-6221. doi:10.5194/acp-8-6199-2008

[10] G. W etzel, A. Bracher, B, F unke, et al., "Validation of MIPAS-ENVISAT $\mathrm{NO}_{2}$ Operat ional Data," Atmospheric Chemistry and Physics, Vol. 7, 2007, pp. 3261-3284. doi:10.5194/acp-7-3261-2007

[11] D. Y. Wang, M. Höpfner, C. Blom, et al., "Validation of MIPAS $\mathrm{HNO}_{3}$ operational data," Atmospheric Chemistry and Physics, Vol. 7, 2007, pp. 4905-4934. doi:10.5194/acp-7-4905-2007

[12] S. C. Müller, N. Kämpfer, D. G. Feist, et al., "Validation of Stratospheric Water Vapour Measurements from the Air-Borne M icrowave Radiom eter AM SOS," Atmospheric Chemistry and Physics, Vol. 8, 2008, pp. 3169-3183. doi:10.5194/acp-8-3169-2008

[13] G. Zhang, "Validation of Target Parameters of ENVISAT Chemistry Instr uments with Co rrelative Ba lloon Observations Obtained b y MIPAS-B," Ph.D. Dis sertation, Karls- ruhe Technology University, Karlsruhe, 2006.

[14] C. D. Rodgers and B. J. C onnor, "Intercomparison of ReMote Sounding Instruments," Journal of Geophysical Research Atmospheres, Vol. 108, 2003, pp. 4116-4130. doi:10.1029/2002JD002299 